\title{
Machine Learning Biometric Attendance System using Fingerprint Fuzzy Vault Scheme Algorithm and Multi- Task Convolution Neural Network Face Recognition Algorithm
}

\author{
Patrick Cerna \\ Department of Information \\ Technology \\ Federal TVET Institute \\ Addis Ababa, Ethiopia
}

\author{
Mary Charlemaine \\ Abas \\ Department of Electronics \\ and Electrical Technology \\ Federal TVET Institute \\ Addis Ababa, Ethiopia
}

\author{
Haftom \\ Gebreziagbher \\ Department of Information \\ Technology \\ Federal TVET Institute \\ Addis Ababa, Ethiopia
}

\author{
Mesay Mengstie \\ Department of Electronics \\ and Electrical Technology \\ Federal TVET Institute \\ Addis Ababa, Ethiopia
}

\begin{abstract}
In many higher education institutions in particular in Africa including Ethiopia proper attendance monitoring is a very important criteria in providing quality to promote student satisfaction. Biometric technology offers an advanced verification for employees used in most schools and companies. A prototype using biometric technology has been proposed to facilitate the recording of the employees' attendance and generate automatically the payroll The prototype utilize Adafruit Fingerprint Fuzzy Vault scheme algorithm through Raspberry $\mathrm{Pi} 3$, Pi Camera for face detection and recognition using Multi-task Convolution Neural Network (MTCNN) Method and output simulated through MATLAB respectively. A user interface module was develop using Visual Basic where fingerprint and face registration is done and during logging with the prototype. The resulting prototype was tested in the non-academic staff of Federal TVET Institute, an institute of higher learning specializing Technical Vocational Education and Training (TVET) offering both undergraduate and postgraduate program in Addis Ababa, Ethiopia.
\end{abstract}

\section{General Terms}

Fingerprint Fuzzy Vault Scheme Algorithm, Multi-Task Convolution Neural Network Face Recognition Algorithm

\section{Keywords}

Biometrics, Fingerprint Authentication, Deep Learning, Face Recognition, Machine Learning

\section{INTRODUCTION}

In many higher education institutions in particular in Africa including Ethiopia proper attendance monitoring is a very important criteria in providing quality to promote student satisfaction. According to Acroprint [1], it is very important to monitor the employee's attendance or time for accurate computation of payroll and to impose discipline to employee with regards to time. Some companies and schools are using a manual punch card to record the employee's attendance and others are still using a logbook. Using a logbook, employees are writing down their names, time and signature to login and logout in the office/school. In the use of a punch card machine, employees are inserting the time card or punch card into a slot on the Bundy clock as they login or logout in the office. This conformed with the study conducted by Harris Interactive Inc. showed that 21 percent of hourly employees admit to stealing company time. While only 5 percent participated in buddy punching, 69 percent said they punch in and out earlier or later than scheduled, 22 percent put additional time on their time sheet, and 14 percent did not punch out for unpaid lunches or breaks [2].

Biometric technology offers an advanced verification for employees used in most schools and companies. Biometric is programmed methods of identify a person or verifying the characteristics of a person based on a physiological or behavioral point. Examples of physiological character include hand or finger images, facial character. Behavioral characters are qualities that are learned or acquire. Dynamic signature authentication, speaker verification and keystroke dynamics are examples of behavioral character. Biometric confirmation requires comparing a registered or enrolled biometric sample beside a newly captured biometric sample for example, a fingerprint captures during a login. During enrollment a sample of the biometric attribute is captured, processed by a computer, and stored for later comparison [3]. This technology involves the identification and verification of individuals by analyzing the human body characteristics and has been widely used in various aspect of life for different purposes. Despite the numerous advantages of the biometric system and its impact to various work sectors across the globe, most users of biometric technology still face the challenge of defining the right and accurate biometric technology system that will be cost effective in solving particular problems in specific environment [4]. The rise in the number of biometrics systems application shows that it is a very promising utilization of technology to provide better results. Its applications can be observed to provide solutions to specific situational needs of those that are using it. The study looks into the implementation of fingerprint and face recognition biometric systems to fit the institute's need for a better system in checking attendance for non-teaching staff or administrative staff in an educational institution.

In dealing with this matter, the researcher conducted a study and develops a prototype using biometric technology to facilitate the recording of the employees' attendance and generate automatically the payroll. This proposed technology minimizes the buddy punching and payroll losses as experienced by the other organizations. The employee uses the fingerprint reader to verify and identify the fingerprint image and record their attendance in the school or company, basis for the generation of payroll. The system generates the daily time record (DTR), computes the tardy and under time of an employee, automates income tax deduction, and manages refunds, allowances (per-diem) and deductions. Taking of attendance is time consuming and it is difficult to 
ascertain the number of students that have made the minimum percentage and thus eligible for exam. Thus, there is a need for a system that would eliminate all of these trouble spots. This study develops a machine learning based attendance system using deep learning algorithms Multi-task Cascaded Convolutional Networks (MTCNN) face detection and Fingerprint authentication fuzzy vault scheme algorithm. The prototype utilizes Adafruit Fingerprint Deep Learning algorithm through Raspberry Pi 3, Pi Camera for face detection and recognition using Multi-task Convolution Neural Network (MTCNN) Method and output simulated through MATLAB respectively. The resulting prototype was tested in the non-academic staff of Federal TVET Institute, an institute of higher learning specializing Technical Vocational Education and Training (TVET) offering both undergraduate and postgraduate program in Addis Ababa, Ethiopia.

\section{RELATED WORKS}

Punitha, K. [5] conducted a study on the Enactment of Face Recognition Algorithm for Attendance System and concluded that Face affirmation can be an assistive system hoping to help multi-measured looking at application as a champion among the most logo additionally, "easy to-assemble" face revelation. EmguCV computation to be utilized completed Attendance System attempt. Curves of EmguCV count's execution bulldozed the Fisher confront computation's execution using the current getting ready set.

Patil et. al [6] conducted a study entitled "A Wireless Fingerprint Attendance System" which combines fingerprint authentication with the process of attendance management and thus forming a novel "automatic attendance management technique". It comprises of three processes namely; enrollment, attendance and reporting. Enrollment process covers capturing the biometrics of a person and storing it in a flash memory against the person's id. The simple aim of the enrollment module is to register the user using his/her id and fingerprints into a flash memory after feature extraction The system consists of fingerprint acquisition module, zigbee transmission and receiving module and attendance management workstation comprises of ARM7 processor.

Chandramoha et. al [7] conducted a study entitled "Attendance Monitoring Systems of Students Based on Biometric and GPS Tracking System" which is fingerprint recognition system based on minutiae based fingerprint algorithms used in various techniques. The system ignores the requirement for stationary materials and personnel for keeping of records. Its objective is to develop an embedded system, which is used for security applications and includes a microcontroller based prototype of attendance system using fingerprint sensor and face recognition module is implemented. In addition, the tracking module is used here to identify the location of the missing person.

Muhammad Fuzail et.al. [8] conducted a study entitled "Face Detection System for Attendance of Class' Students", An regular attendance supervision system is a essential tool for any LMS. Most of the existing system are time consuming and necessitate for a semi instruction manual work from the instructor or students. This approach aim to explain the issues by integrates face detection in the procedure. Even though this method still lacks the capability to identify each student in attendance on class, there is still much more room for enhancement. Another issue that has to be taken in consideration in the opportunity is a process to ensure users privacy. Whenever you like a representation is stored on servers, it must be impossible for a person to use that image.
Mathana Gopala et. al. [9] conducted a study entitled "Implementation of Automated Attendance System using Face Recognition", an automated presence System has been envision for the purpose of falling the errors that occur in the conventional (manual) attendance taking system. The aim is to computerize and make a system that is useful to the institute such as an organization. The efficient and exact method of attendance in the office atmosphere can reinstate the old manual methods. This technique is secure enough, reliable, and available for use. No need for dedicated hardware for installing the system in the office. It can be constructed using a camera and computer.

S. B. Dabhade et. al. [10] conducted a study entitled "Face Recognition using Principle Component Analysis and Linear Discriminate Analysis Comparative Study" recognition Rate some time enlarged sometime reduce, sometime stable. The research have done various research like particular image for enrolment and particular image for testing, then had keep the enrolment image as constant and change testing images such as two, three, four, up to nine. For taking the consequence the research had used three databases ORL Database, KVKRFace Database and IIT-Indian Database.

\section{MATERIALS AND METHODS 3.1 Block Diagram}

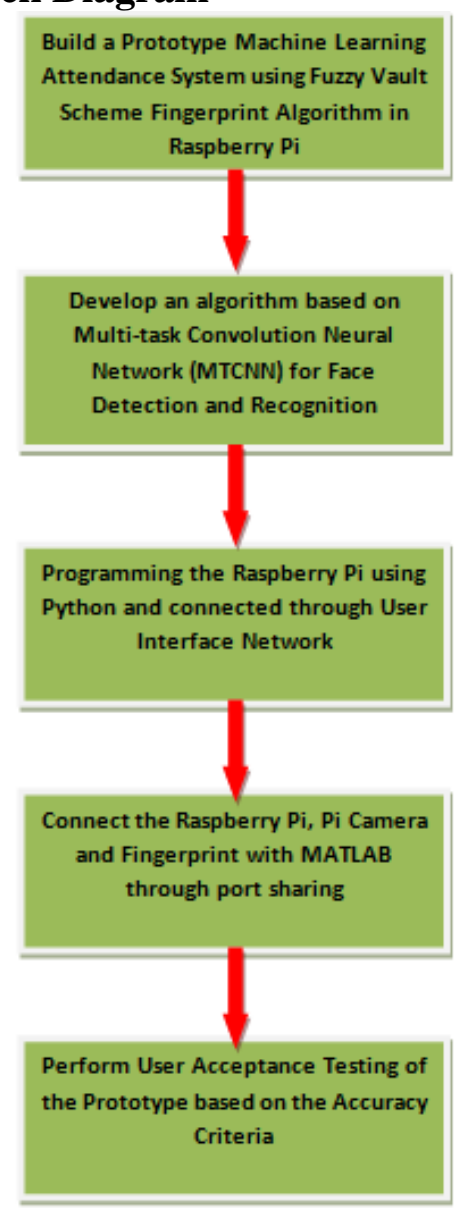

Figure 1: System Flow Chart

Figure 1 presents the phases of principal tasks representing the progression of the project towards the end goal of developing and implementing a fingerprint and facial recognition biometric system for attendance checking. The first phase is the development of $\mathrm{p}$ machine learning fingerprint prototype adopting Fuzzy Vault Scheme algorithm 
followed by the second phase incorporating the Face Detection and Recognition using Multi-task convolution neural network algorithm in Raspberry $\mathrm{Pi}$ and Pi Camera respectively.

Python programming language is used to program the Raspberry Pi and connected through user interface network. After this, the biometric reader Raspberry pi, and Pi Camera is then connected with the algorithm and output is simulated to MATLAB through port sharing. This includes the creation of MATLAB algorithm that will be used for the management of biometric data from camera for face recognition.

\subsection{Fingerprint}

A fingerprint is obtained from the friction ridges of the finger. High and peaking part of the skin causes the dark lines in the fingerprint as it is shown in Figure 2. White spaces in between dark lines are due to the shallow parts of the skin, which are also called the valleys. The ridges and furrows enable us to firmly hold objects. Their presence causes a friction, which is needed to grab any object. But uniqueness of fingerprint is not due to these ridges and furrows. Uniqueness is achieved due to minutiae points. The combination of minutiae points of two different fingers of an individual enables privacy protection in [11].

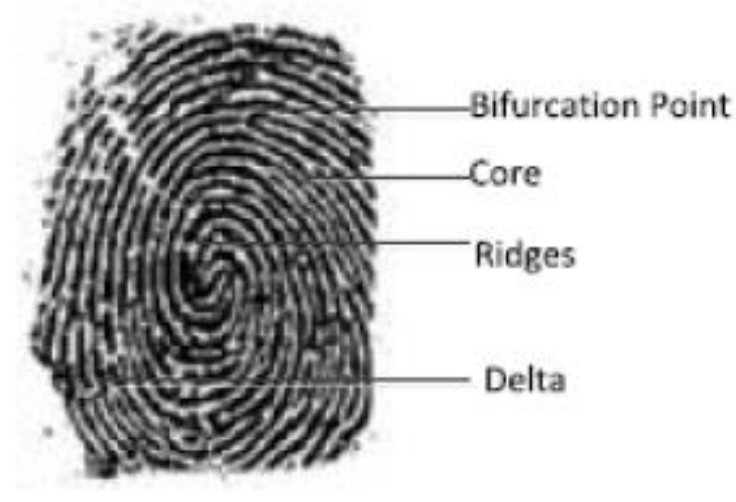

Figure 2. Fingerprint

\subsection{Biometric Authentication and \\ Processing}

Biometric authentication involves the completion of six phases as reflected in Figure 3. The registration phase includes pre-processing, region of-interest detection and feature extraction steps. The extracted features are then stored in the database. The recognition phase includes preprocessing, region-of-interest detection, feature extraction, matching and decision making steps.

\subsubsection{Finding Region of Internet (ROI)}

Locating the Region of Interest (ROI) for a biometric trait is essential precursor for feature extraction step. This step identifies the main or interesting portion of the image (or signal) from where the biometric traits are extracted. The techniques to identify the region of interest can be grouped into three major divisions, namely Bottom-Up Feature Based Approach, Top-Down Knowledge Based Approach through individual's motion and appearance in determining the region of interest [12] and Appearance Based Approach on how region of interest of a palm is extracted [13].

\subsubsection{Feature Extraction}

Two different feature extraction approaches are present for handwritten signatures as they aim to capture the static or the dynamic features. Geometrical features of the signature are considered for the static approach. A dynamic feature of handwritten signature includes the speed and the acceleration of the pen movement, penup and pen-down times, etc. Ear curves are extracted for an ear recognition system in [14]. The face recognition system in [15] has used the techniques like wavelet transform, spatial differentiation and twin pose testing scheme for feature extraction from faces. According to [Ukpai et al, 2015], principal texture pattern and dual tree complex wavelet transform produce iris-specific features from an iris image. The next section on various biometric traits will lead to a better understanding of this through narration of different biometric traits.

\subsubsection{Matching and Decision}

In this step, the extracted features are compared with the enrolled features to obtain a matching score. The subsequent decision making step either accepts or rejects an individual using this matching score.

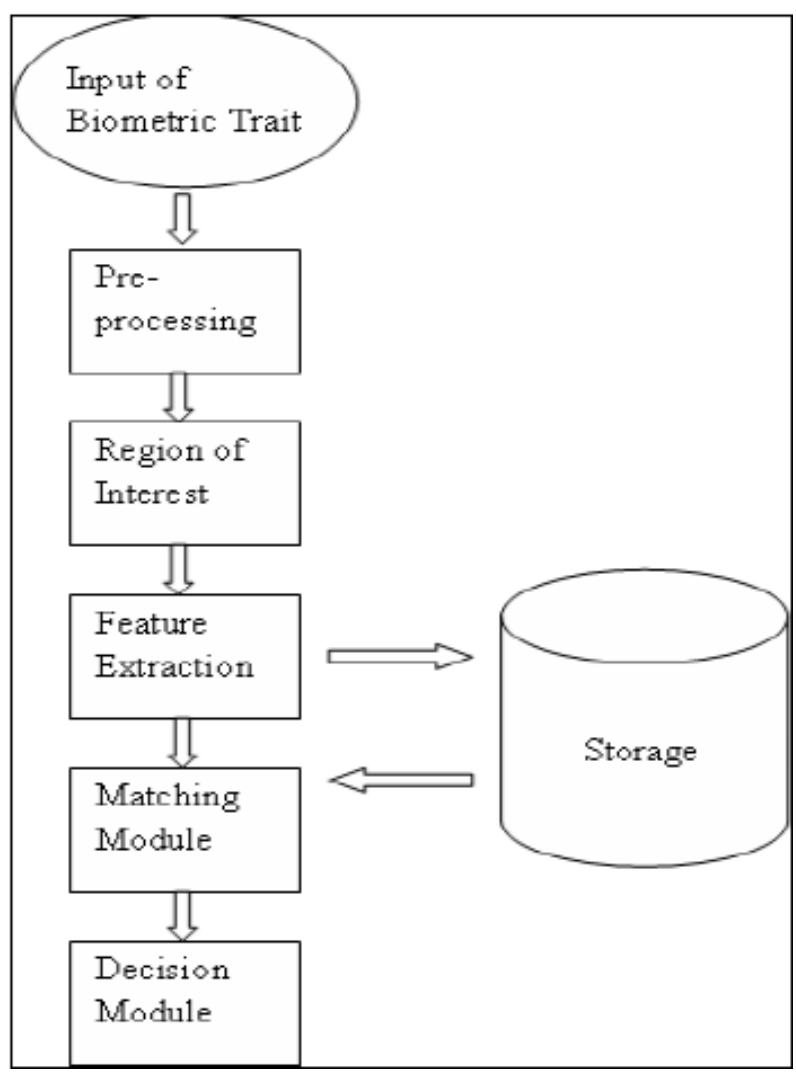

Figure 3: Phases of Biometric Authentication

\subsubsection{Fuzzy Vault Scheme Fingerprint Algorithm}

Juels and Sudan [16] originally proposed Fuzzy vault scheme for which security is based on the infeasibility of the polynomial reconstruction problem. The fuzzy vault scheme stores only a transformed version of the template, which makes it applicable to various modalities besides fingerprints [17] [18]. Given two or more such fuzzy vault instances generated from the same point, but with different keys and different random chaff, the minutiae are likely recoverable by matching the two templates [19]. If an attacker is able to recover the secret $\mathrm{k}$ through means other than attack against the template it becomes trivial to recover biometric data. From secret, polynomial is directly reconstructed and hence biometric can be achieved [20]. This is reflected in Figure 4 showing the authentication mechanism using Fuzzy Vault Scheme Fingerprint Algorithm. 


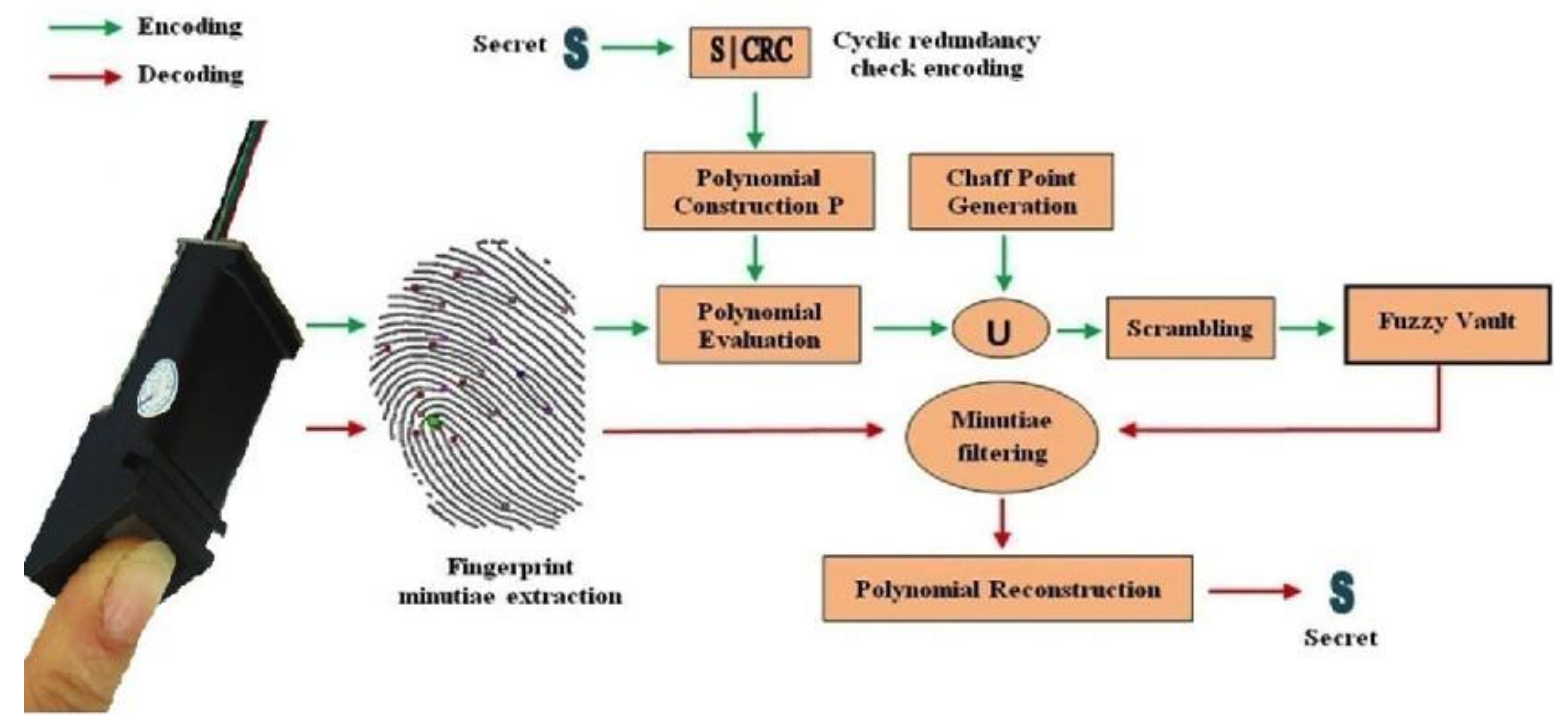

Figure 4: Fuzzy Vault Scheme Fingerprint Algorithm

\subsection{Face Recognition Algorithm}

Deep learning is a subfield of machine learning, which aims to learn a hierarchy of features from input data. Nowadays, researchers have intensively investigated deep learning algorithms for solving challenging problems in many areas such as image classification, speech recognition, signal processing, and natural language processing. Deep Learning algorithms are quite beneficial when dealing with learning from large amounts of unsupervised data, and typically learn data representations in a greedy layer-wise fashion [21,22].

One of this algorithm is Multi-task Convolution neural network (MTCNN) is one of the most powerful classes of deep neural networks in image processing tasks. It is highly effective and commonly used in computer vision applications. The convolution neural network contains three types of layers: convolution layers, sub-sampling layers, and full connection layers
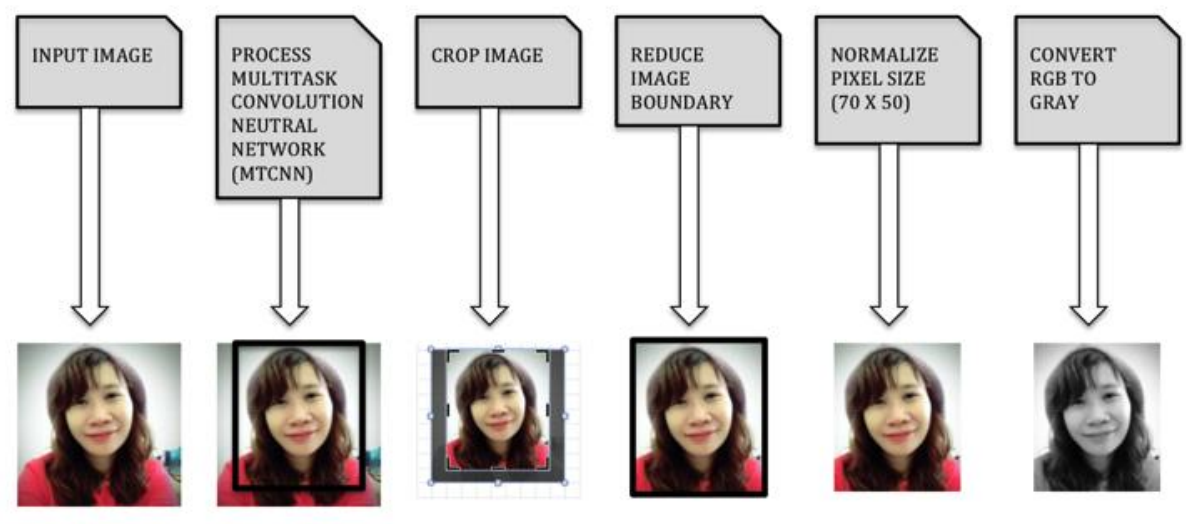

Figure 5: Face Recognition and Processing

In this study, the six phases of face detection and processing is reflected in Figure 5 using MTCNN.

1. Face Detection. This part is where the computer locates the main face in the captured image. An image with no face detected will be considered an unsuccessful input and therefore will go back to accepting fingerprint data for identification.

2. Face Processing. It is the process where the located face is processed and adjusted to become clear and more similar to other faces. The face image is also converted into grayscale. This process correlates to the process of adjusting the image size from the camera and image processing for comparison. This process where the image further cropped and resized is called localization while the process where the image is converted into grayscale is called normalization.
3. Storing Data. It is the process where the processed face images are stored in the database and then learned by the computer for future referencing. These images are grayscale 70 x 55 pixels. Number of sample per person can be varied upon enrolment of subject

4. Face Recognition. It is the process that checks which of the stored processed face images in the database is the most similar to the face in the camera. In this design, the researcher adopt the Multi-task Convolution neural network (MTCNN) to compare processed images

\subsection{Operation}

The operation of the system for attendance checking starts when an administrative username and password is entered as reflected in Figure 6. First stage of the biometric checking is 
the fingerprint identification. The process will never proceed to face detection and recognition unless a fingerprint in the database is observed as an input. On the face detection and recognition stage, a matched image and matched fingerprint will be recorded as an attendance and an unrecognized image even with matched fingerprint will not be recorded as attendance and will proceed to start again where the system waits for an input fingerprint.

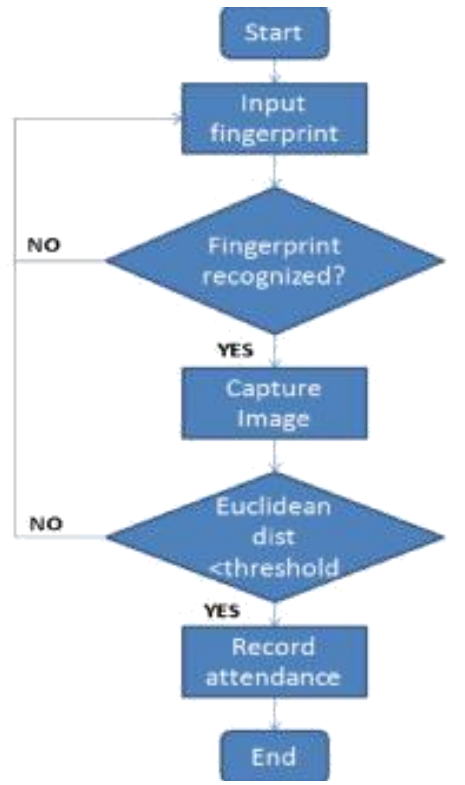

Figure 6: System Flow Chart

\section{EXPERIMENTAL RESULTS}

The developed prototype has been testing among ten (10) non-teaching staff of Federal TVET Institute, a higher learning institution in Ethiopia. Figure 7 shows the user interface design developed using Visual Basic 2012 with MS SQL Database integrated Raspberry Pi 3, Pi Camera for face detection and Adafruit Fingerprint Sensor.

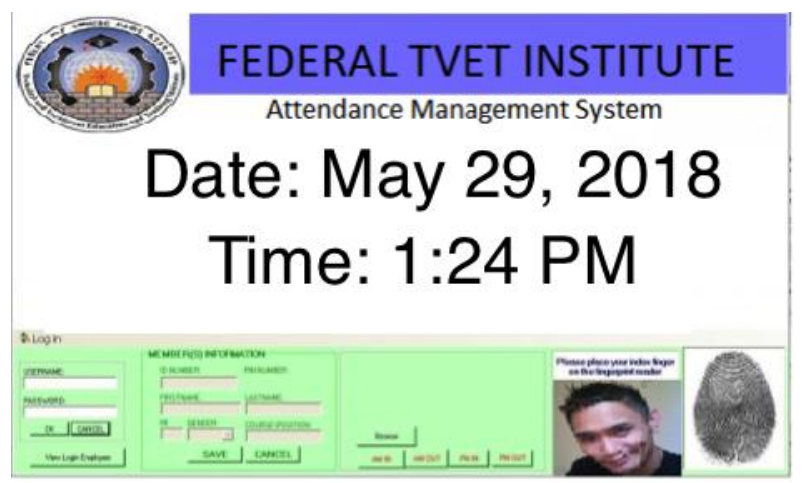

Figure 7: Main User Interface Design

Table 1 shows the results of the fingerprint authentication with all the five (5) type of fingers administered to ten (10) employees. The result implies that the system produce an accuracy of greater than 90 percent based several trials with 10 samples in the database.

Table 1: Fingerprint Authentication Testing Result

\begin{tabular}{|l|l|l|l|l|l|l|}
\hline Employee & \multirow{2}{*}{$\begin{array}{l}\text { Finger } \\
\text { Name }\end{array}$} & \multicolumn{2}{|l|}{ Finger type and Results } \\
\cline { 3 - 7 } & & Thumb & Pointing & Middle & Ring & Index \\
\hline Berhanu & 101 & Yes & Yes & Yes & No & Yes \\
\hline Hana & 102 & Yes & Yes & Yes & Yes & Yes \\
\hline
\end{tabular}

\begin{tabular}{|l|l|l|l|l|l|l|}
\hline Selamawit & 103 & Yes & No & Yes & Yes & Yes \\
\hline Habtamu & 104 & Yes & Yes & Yes & Yes & Yes \\
\hline Tewodros & 105 & Yes & Yes & Yes & Yes & Yes \\
\hline Mengistu & 106 & Yes & Yes & No & Yes & Yes \\
\hline Firaol & 107 & Yes & Yes & Yes & No & Yes \\
\hline Meseret & 108 & Yes & Yes & Yes & Yes & Yes \\
\hline Daniel & 109 & Yes & Yes & Yes & Yes & Yes \\
\hline Genene & 110 & Yes & Yes & Yes & Yes & No \\
\hline
\end{tabular}

The Euclidean distance threshold of 100,000 specifies that an image during face recognition will still be considered a match if the difference between their eigenvectors is lower than 100,000 . Table 2 shows the portion of the results taken from five (5) trials of Fingerprint ID 101 measuring the Euclidean distance based on the correct matches and incorrect matches. Euclidean distance Mean has been computed by averaging both the correct and incorrect matches for 5 trials with value 40,011 for Fingerprint ID 101.

Table 2: Trials for Finger ID 101 Euclidean Distance

\begin{tabular}{|l|l|l|l|}
\hline \multirow{2}{*}{ Fingerprint ID } & Trial & $\begin{array}{l}\text { Euclidean Distance } \\
\text { Correct } \\
\text { Match }\end{array}$ & $\begin{array}{l}\text { Incorrect } \\
\text { Match }\end{array}$ \\
\hline 101 & 1 & 28657 & \\
\hline 101 & 2 & 25959 & \\
\hline 101 & 3 & & 53784 \\
\hline 101 & 4 & 50382 & \\
\hline 101 & 5 & 41276 & \\
\hline
\end{tabular}

Figure 8 shows the Euclidean Distance Mean for each of Fingerprint ID. Euclidean distances of successfully matched images, values of average Euclidean distances vary from 40,011 to 59,172 .

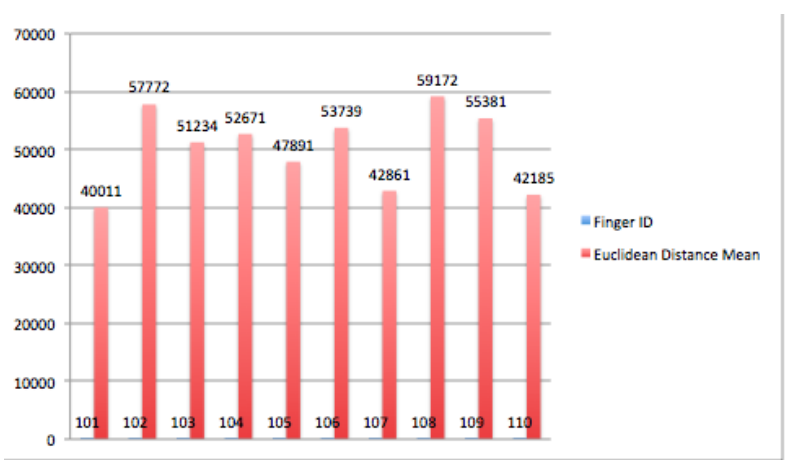

Figure 8: Face Recognition Euclidean Distance Mean

\section{CONCLUSION}

The study resulted in a prototype system utilizing Adafruit Fingerprint Fuzzy Vault scheme algorithm through Raspberry Pi 3, Pi Camera for face detection and recognition using Multi-task Convolution Neural Network (MTCNN) Method and output simulated through main user interace respectively.

The biometric fingerprint authentication generates a very high percent accuracy. However, it is observed by the researchers that the unrecognized fingerprint data input are mainly from the finger not being pressed hard on the scanner. Maximum Euclidean distance is determined through the average of computed. 


\section{RECOMMENDATION}

The researcher recommends that to enhanced the study by utilizing other hardware component e.g. Arduino microcontroller, and other peripherals to test the algorithm used for fingerprint authentication and face recognition. It is also recommended that user interface design must support mobile application integrated with current prototype running in a distributed system through service-oriented architecture (SOA).

\section{REFERENCES}

[1] Acroprint 2018. Why Employees (Should) Love Time and Attendance. Retrieved from https://www.acroprint.com/newsletter-archives/201210.php. Accessed February 2018.

[2] Gale, F. S. 2013. Employers Turn to Biometric Technology to Track Attendance. Retrieved from http://www.workforce.com/2013/03/05/employers-turnto-biometric-technology-to-track-attendance/. Accessed February 2018.

[3] K.Senthamil Selvi, P.Chitrakala, A.Antony Jenitha, Face recognition based Attendance marking system IJCSMC, Vol. 3, Issue. 2, February 2014, 337 - 342

[4] Oloyede MO, Adedoyin AO, Adewole KS (2013) Fingerprint Biometric Authentication for Enhancing Staff Attendance System. International Journal of Applied Information System 5: 19-24.

[5] Punitha, K. 2017. Enactment of Face Recognition Algorithm for Attendance System. International Journal of Applied Engineering Research. Vol 12, Issue. 4.

[6] Patil, P., Khachane, A and Purohit, V. 2016. A Wireless Fingerprint Attendance System. International Journal of Security, Privacy and Trust Management. Vol 5, Issue 4

[7] Chandramohan, J, Nagarajan R., Kumar, M., Dineshkumar, T., Kannan, G., and Prakash, R. International Journal of Advanced Engineering, Management and Science, Vol 3, Issues 3

[8] Muhammad Fuzail, Hafiz Muhammad Fahad Nouman, Muhammad Omer Mushtaq, Binish Raza, Awais Tayyab, Muhammad Waqas Talib 2014. Face Detection System for Attendance of Class' Students. International Journal of Multidisciplinary Sciences and Engineering, Vol. 5, Issue 4.

[9] Mathana Gopala Krishnan, Balaji, Shyam Babu 2015. Implementation of Automated Attendance System using Face Recognition. International Journal of Scientific \& Engineering Research. Volume 6, Issue 3

[10] S. B. Dabhade, Y. S. Rode, M. M. Kazi, R. R. Manza and K. V. Kale 2013. Face Recognition using Principle Component Analysis and Linear Discriminant Analysis

[11] Comparative Study. 2nd National Conference on Advancements in the Era of Multi-Disciplinary Systems

[12] S. Li and A. C. Kot 2013. Fingerprint Combination for Privacy Protection. IEEE Transactions on Information Forensics and Security, Vol. 8, No. 2, 350- 360
[13] M. J. Jones and P. Viola 2006. Method and System for Object Detection in Digital Images. US Patents 7099 510.

[14] A. Saliha, B. Karima, K. Mouloud, D. H. Nabil, and B. Ahmed 2014. ExtractionMethod of Region of Interest from Hand Palm: Application with Contactless and Touchable Devices. 10th International Conference on Information Assurance and Security (IAS), 77-82.

[15] L. Ghoualmi, A. Draa, and S. Chikhi, 2015. An Efficient Feature Selection Scheme Based on Genetic Algorithm for Ear Biometrics Authentication. 12th International Symposium on Programming and Systems (ISPS), pp. 1-

[16] A. Saliha, B. Karima, K. Mouloud, D. H. Nabil, and B. Ahmed 2014. Extraction Method of Region of Interest from Hand Palm: Application with Contactless and Touchable Devices," in Proc. 10th International Conference on Information Assurance and Security (IAS), 77-82.

[17] Ari Juels and Madhu Sudan 2002. A Fuzzy Vault Scheme. IEEE International Symposium Information Theory, Lausanne, Switzerland, 408, 2002.

[18] Clancy, D Lin and N Kiyavash 2003. Secure smartcardbased fingerprint authentication. Proceedings of ACM SIGMM Workshop on Biometric Methods and Applications, Berkley, CA, 45-52

[19] Cengiz Orencik 2008. Fuzzy Vault Scheme for Fingerprint Verification: Implementation, Analysis and Improvements. Thesis. Sabanci University

[20] Hoi Ting Poon and Ali Miri 2008. A Collusion Attack on the Fuzzy Vault Scheme", University of Ottawa, ISC, 27-34.

[21] Sumin Hong, Woongryul Jeon, Seungjoo Ki, Dongho Won, Choonsik Park (2008). The Vulnerabilities Analysis of Fuzzy Vault using Password. Proceedings of IEEE, Vol. 3, 76-83.

[22] Hinton GE, Osindero S, Teh Y-W 2006. A fast learning algorithm for deep belief nets. Neural Comput 18(7): $1527-1554$

[23] Sannella, M. J. 1994. Constraint Satisfaction and Debugging for Interactive User Interfaces. Doctoral Thesis. UMI Order Number: UMI Order No. GAX9509398., University of Washington.

[24] Forman, G. 2003. An extensive empirical study of feature selection metrics for text classification. J. Mach. Learn. Res. 3 (Mar. 2003), 1289-1305.

[25] Brown, L. D., Hua, H., and Gao, C. 2003. A widget framework for augmented interaction in SCAPE.

[26] Y.T. Yu, M.F. Lau, "A comparison of MC/DC, MUMCUT and several other coverage criteria for logical decisions", Journal of Systems and Software, 2005, in press.

[27] Spector, A. Z. 1989. Achieving application requirements. In Distributed Systems, S. Mullender. 